\title{
The second realization of the ICRF with VLBI
}

\author{
C. $\mathrm{Ma}$
}

NASA Goddard Space Flight Center

Code 698, Greenbelt, Maryland, 20771, USA

email: Chopo.Ma@nasa.gov

\begin{abstract}
The ICRF derived from VLBI observations of extragalactic radio sources up to 1995.6 and effective since 1998.0 was a radical change from the FK5 stellar/equinox celestial system. Since then the number of geodetic/astrometric VLBI observations has tripled and the number of radio sources with astrometrically useful data has quadrupled. These data along with advances in modeling and estimation will be used to generate the next ICRF realization in the microwave band. Analysis of source position time series and source structure evolution will be used to select better "defining" sources. Working groups have been established by the IAU, IERS and IVS with the goal of presenting the second realization of the ICRF at the IAU General Assembly in 2009 .
\end{abstract}

Keywords. : astrometry, reference systems, catalogs, surveys

\section{Introduction}

Since 1998 the basis for celestial positioning has been the ICRS (International Celestial Reference System) and the ICRF (International Celestial Reference Frame). The following descriptions are taken from the NFA IAU 2000 Glossary developed by the IAU Working Group "Nomenclature for Fundamental Astronomy" (Capitaine et al. (2007)):

The ICRS is the idealized barycentric coordinate system to which celestial positions are referred. It is kinematically nonrotating with respect to the ensemble of distant extragalactic objects. It has no intrinsic orientation but was aligned close to the mean equator and dynamical equinox of J2000.0 for continuity with previous fundamental reference systems. Its orientation is independent of epoch, ecliptic or equator and is realized by a list of adopted coordinates of extragalactic sources.

The ICRF is a set of extragalactic objects whose adopted positions and uncertainties realize the ICRS axes and give the uncertainties of the axes. It is also the name of the radio catalog whose 212 defining sources are currently the most accurate realization of the ICRS. Note that the orientation of the ICRF catalog was carried over from earlier IERS radio catalogs and was within the errors of the standard stellar and dynamical frames at the time of adoption. Successive revisions of the ICRF are intended to minimize rotation from its original orientation. Other realizations of the ICRS have specific names (e.g., the Hipparcos Celestial Reference Frame).

The ICRF catalogue was generated from $2 / 8 \mathrm{GHz}$ VLBI data and the analysis available in mid 1995. The error for individual source positions has an estimated floor of 250 
microarcseconds and the accuracy of the realized ICRS coordinate axes is $\sim 30$ microarcseconds based on the 212 defining sources. The ICRF was updated to ICRF-Ext.2 (Fey et al. (2004)) using additional data through 2002, but the axes are unchanged since the same defining sources and their ICRF positions are retained.

\section{Organization of the second VLBI realization}

The ICRS/ICRF was adopted by a resolution of the IAU General Assembly in Kyoto in 1997 acting for the whole astronomical community. The groundwork had been laid through a series of IAU working groups and colloquia held over nearly a decade that addressed both the relativistic foundation and the suitability of compact extragalactic radio sources as fiducial objects. The maintenance of the ICRS/ICRF was delegated by the IAU to the IERS (International Earth Rotation and Reference Systems Service). As the ICRF was derived from VLBI observations, the IVS (International VLBI Service for Geodesy and Astrometry), one of the independent Technique Centers of the IERS, has the primary responsibility for continuing observations and analysis to support advancement of the ICRF at radio frequencies. There are two working groups involved in the second ICRF realization, one established by the IAU to provide oversight for the wide astronomical community and the other established jointly by the IERS and IVS to carry out the actual analysis. The charter of the IERS/IVS Working Group states:

The purpose of the working group is to generate the second realization of the ICRF from VLBI observations of extragalactic radio sources, consistent with the current realization of the ITRF and EOP data products. The working group will apply state-of-the-art astronomical and geophysical models in the analysis of the entire relevant S/X astrometric and geodetic VLBI data set. The working group will carefully consider the selection of defining sources and the mitigation of source position variations to improve the stability of the ICRF. The goal is to present the second ICRF to relevant authoritative bodies, e.g. IERS and IVS, and submit the revised ICRF to the IAU Division I working group on the second realization of the ICRF for adoption at the 2009 IAU General Assembly.

\section{VLBI observations to improve the ICRF}

One difficulty faced by the original ICRF analysis was the fact that the overall VLBI data set was $\sim 95 \%$ from geodetic observing sessions, which used a limited set of "geodetic" sources. The geodetic sources are generally the strongest "compact" sources, including in the early years of geodetic/astrometric VLBI some sources with considerable structure like 3C273B. Figure 1 shows that the imbalance between the number of sessions for geodetic and non-geodetic sources is still present. However, the peak at 11-30 sessions reflects a specific IVS observing program to enhance the astrometric data set based on Feissel-Vernier's analysis of annual source position time series (Feissel-Vernier (2003)), which identified sources with stable and unstable positions. Figure 2 shows stable and potentially stable sources separated between ICRF defining and non-defining sources. Potentially stable sources did not have sufficient data in 1989-2002 to fully categorize stability but had no indication of instability. Figure 3 shows that the current set of ICRF defining sources is probably not ideal, some being unstable and some having minimal data during 1989-2002. The stable, potentially stable and non-geodetic ICRF defining sources have been observed regularly since 2004 in the IVS CRF monitoring program, which uses a small fraction of the time during selected geodetic observing sessions (Fig. 4). The goal 
is to observe each source at least semiannually. The data will be used in the selection of defining sources for the second realization. Figures 5 and 6 show that the CRF monitoring sources and the ICRF defining sources have been observed, but much more data would be desirable. Southern sources are observed in specific astrometric sessions, but the networks are small.

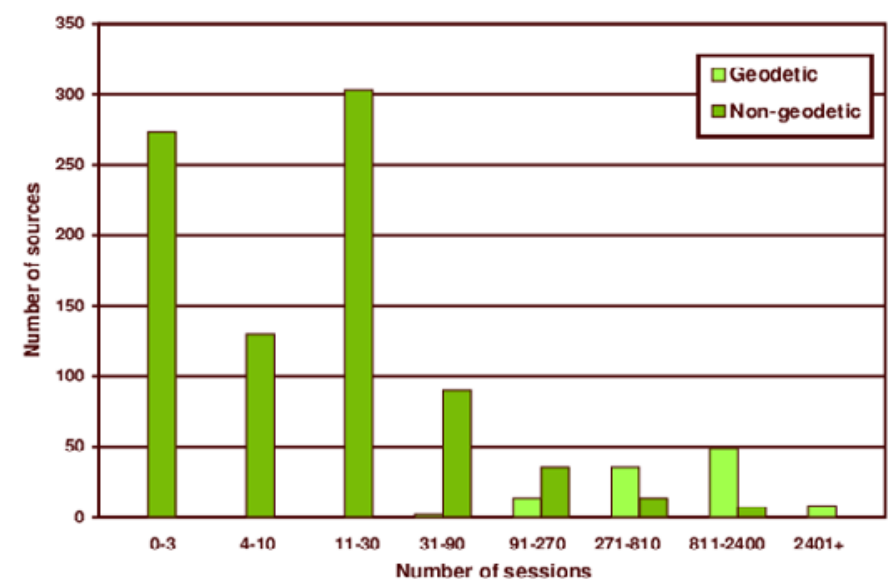

Figure 1. Sources' session participation, 1979-2007.

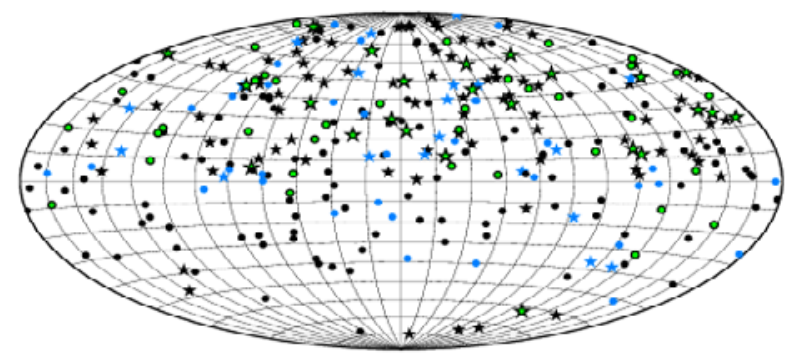

Defining: $\star 81$ Stable: F..V.AKA $2003 \star 20$ Stable: F..V. additional 25 Potentially stable Other: - 117 Stable: F.-V.A\&A 2003 33 Stable: F.-V.additional $\bullet 36$ Potentially stable

Figure 2. Stable and potentially stable sources.

\section{Position time series and source structure}

Position time series will be one of the means for selecting the defining sources for the next VLBI ICRF. New time series have been generated by members of the IERS/IVS Working Group for this purpose. Figure 7 shows the sum of the wrms scatter for the right ascension and declination time series as a vector at the position of each source. Only sources with summed wrms $<0.5$ mas are included. Although there are probably enough sources to select good defining sources, it can be seen that there is a data deficiency below $-20^{\circ}$ declination in both quantity and quality. This deficiency may require flexible criteria for defining sources to achieve more uniform spatial distribution.

Source structure index information will also be used to select the defining source (See Charlot, this volume). Structure index 1 and 2 indicate minimal structure while index 3 

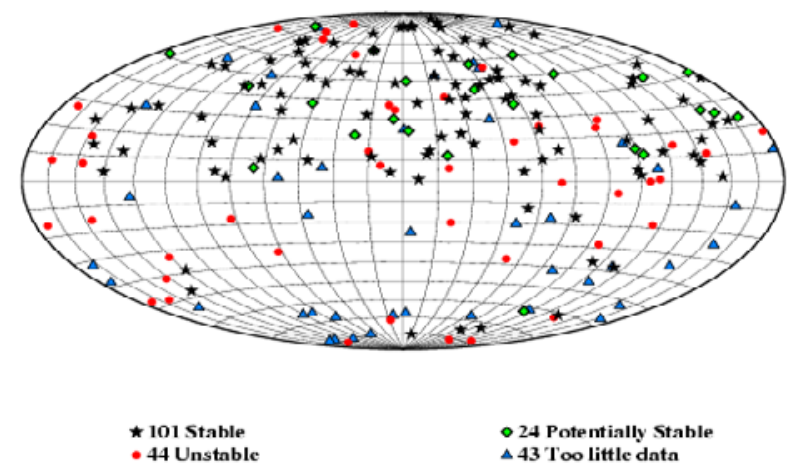

Figure 3. ICRF defining sources.

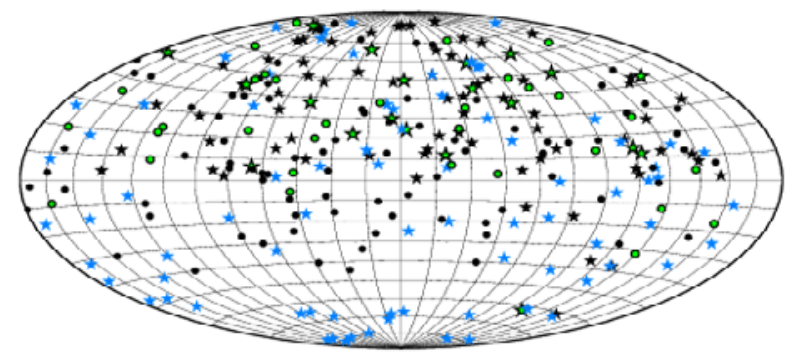
$\begin{array}{ll}\star 74 \text { Stable ICRF } & \$ 25 \text { Potentially stable ICRF } \\ \bullet 89 \text { Stable other } & \bullet 36 \text { Potentially stable other }\end{array}$

Figure 4. Total CRF monitoring sources.

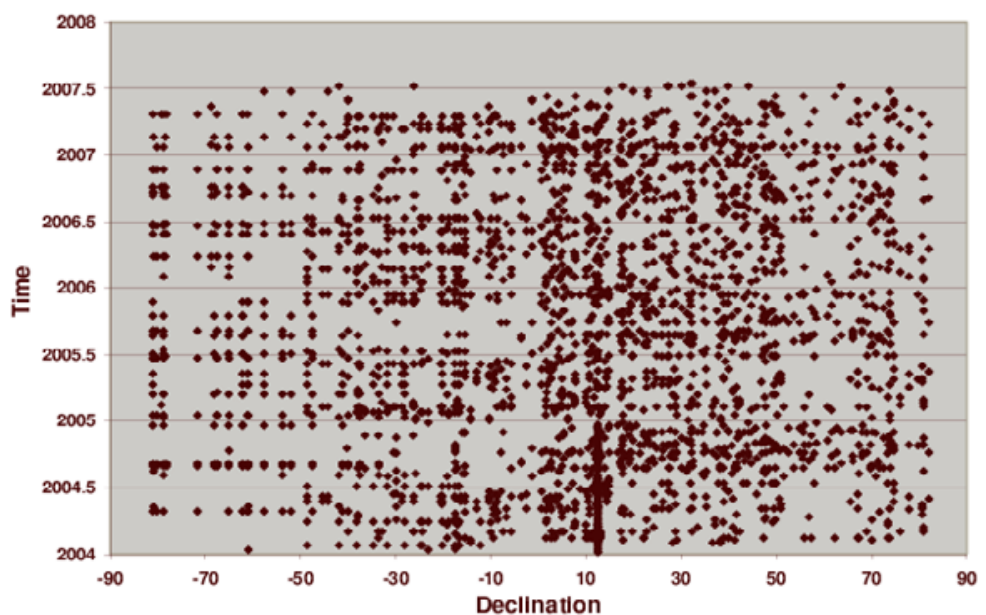

Figure 5. Observations of CRF Monitoring Sources, 2004-2007.

and 4 reflect undesirable complexity. Figures 8 and 9 show there is a relationship between structure index and scatter of position time series. Index 1 and 2 sources generally have less scatter than index 3 and 4 sources. There are, however, outliers that need to be examined. 


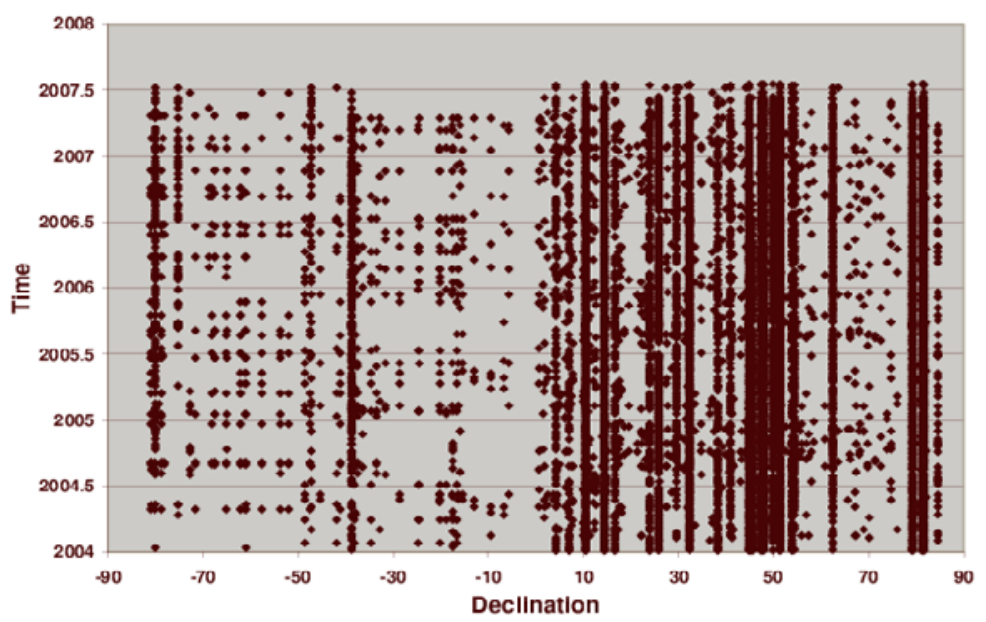

Figure 6. Observations of ICRF Defining Sources, 2004-2007.

Sources (285) with Arc WRMS $<0.5$ mas

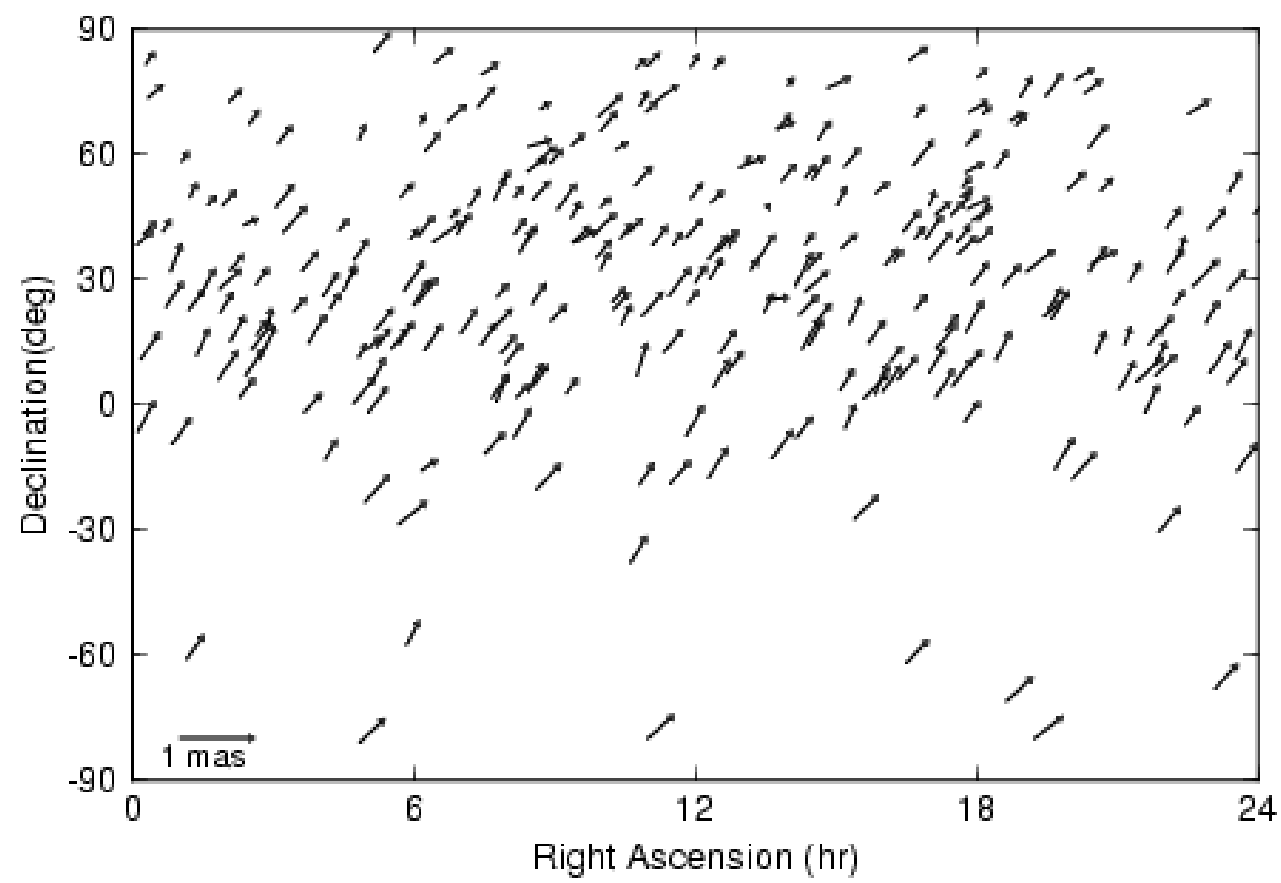

Vector components are [wrms(RAcosDec), wrms(DEC)]

Figure 7. Summed wrms scatter (for sums $<0.5$ mas only). 


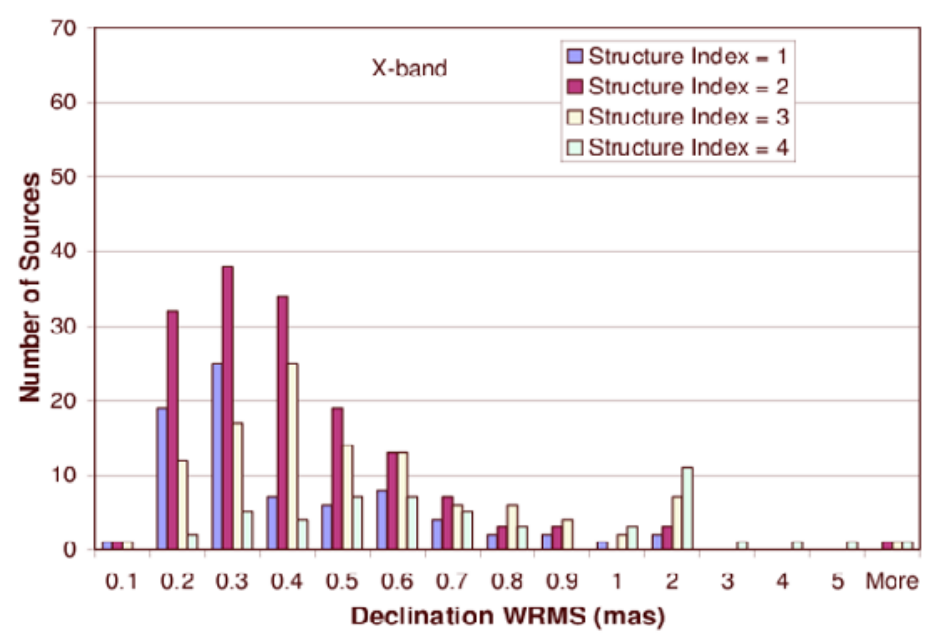

Figure 8. Distribution of sources for declination scatter (categorized by structure index).

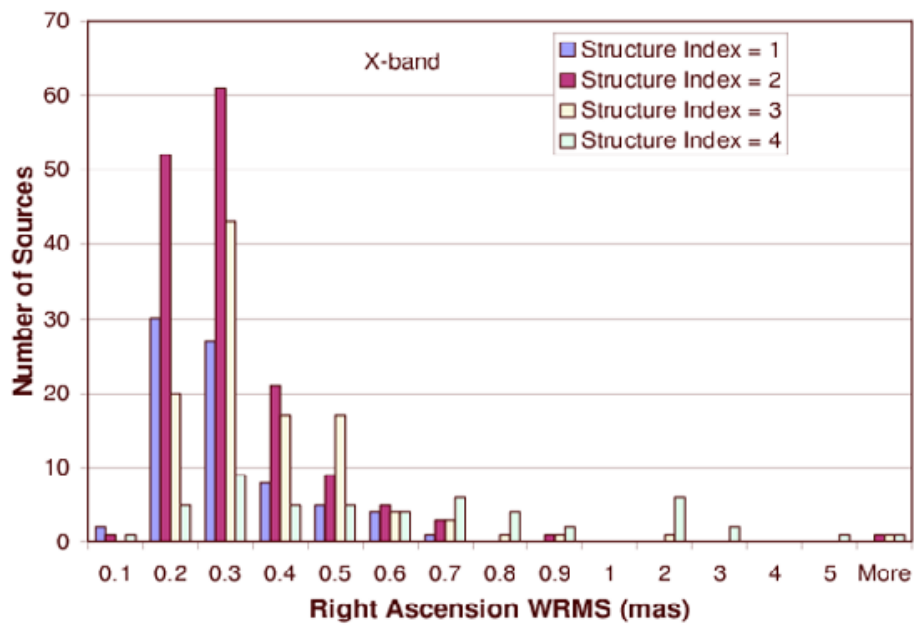

Figure 9. Distribution of sources for right ascension scatter (categorized by structure index).

\section{Timetable for IERS/IVS Working Group}

\begin{tabular}{|l|l|}
\hline Spring 2007 & Working Group meeting, Vienna \\
\hline Fall 2007 & $\begin{array}{l}\text { Generation, comparison of time series } \\
\text { Working Group meeting, Paris }\end{array}$ \\
\hline Spring 2008 & $\begin{array}{l}\text { Analysis of time series } \\
\text { Working Group meeting, St. Petersburg }\end{array}$ \\
\hline Mid 2008 & Defining source criteria \\
\hline Fall 2008 & Selection of defining sources, analysis configuration \\
\hline Spring 2009 & $\begin{array}{l}\text { Generation of ICRF-2 catalogue, presentation to IVS, IERS, } \\
\text { and IAU working group }\end{array}$ \\
\hline
\end{tabular}




\section{VLBA Calibrator Survey (VCS)}

In addition to the geodetic and astrometric sessions scheduled by the IVS and earlier international space geodesy collaborations, a series of observations which began in 1994 using the VLBA has provided the much larger VCS catalogue (Beasley et al. (2002), Fomalont et al. (2003), Petrov et al. (2005), Petrov et al. (2006), Kovalev et al. (2007)) with good astrometric positions (Figure 10). Each target source, however, is observed in only one session, so the quality of positions is somewhat worse than from the IVS data set and there is no temporal variation information. These sources will be included in the analysis for the second ICRF realization.

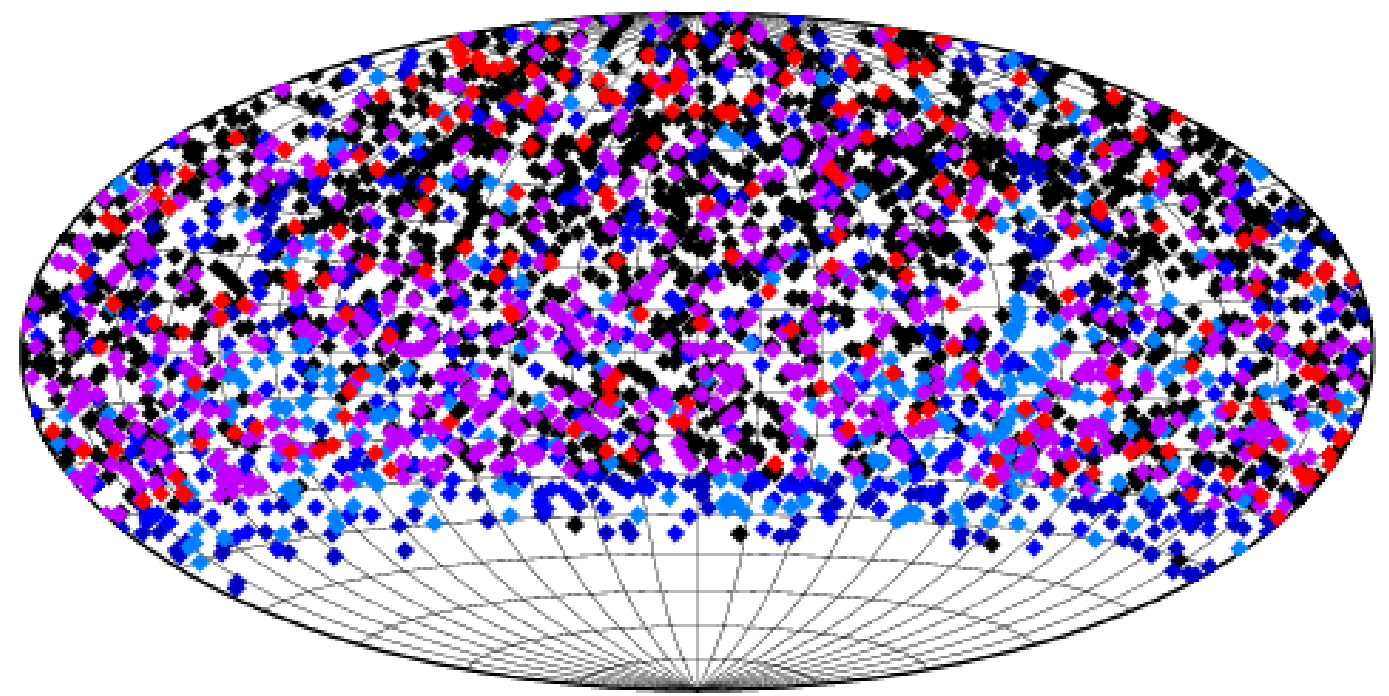

\section{$\bullet 1576$ VCS1 $\bullet 241$ VCS2 $\bullet 308$ VCS3 $\bullet 261$ VCS4 $\bullet 590$ VCS5 $\bullet 215$ VCS6}

Figure 10. VCS sources.

\section{References}

Beasley, A. J., Gordon, D., Peck, A. B., Petrov, L., MacMillan, D. S., Fomalont, E. B., \& Ma, C. 2002, ApJ, Supp. 141, 13

Capitaine et al., IAU Working Group "Nomenclature for Fundamental Astronomy" 2007, http://syrte.obspm.fr/iauWGnfa/

Feissel-Vernier, M. 2003, A\&3A, 403, 105

Fey, A. L., Ma, C., Arias, E. F., Charlot, P., Feissel-Vernier, M., Gontier, A.-M., Jacobs, C. S., Li, J., \& MacMillan, D. S. 2004, AJ, 127, 3587

Fomalont, E., Petrov, L., MacMillan, D. S., Gordon, D., \& Ma, C. 2003, AJ, 126 (N5), 2562

Kovalev, Y., Petrov, L., Fomalont, E., \& Gordon, D. 2007, AJ, 133, 1236

Petrov, L., Kovalev, Y., Fomalont, E., \& Gordon, D. 2005, AJ, 129, 1163

Petrov, L., Kovalev, Y., Fomalont, E., \& Gordon, D. 2006, AJ, 131, 1872 